\title{
Classificazione, diagnosi ed ICD-10. II - Le sindromi schizofreniche
}

\author{
ELISABETTA PALTRINIERI ${ }^{1}$ e GIOVANNI de GIROLAMO ${ }^{2}$ \\ 1 Servizio di Psicologia Medica, Istituto di Psichiatria, Università di Verona, Verona \\ 2 Division of Mental Health, World Health Organization, Geneva
}

\begin{abstract}
Riassunto. Scopo: Presentare un breve profilo storico relativo ai numerosi tentativi di classificazione dei disturbi schizofrenici succedutisi dal secolo scorso ad oggi e descrivere le principali caratteristiche della classificazione ICD-10 della schizofrenia e dei disturbi ad essa correlati, le principali novità introdotte in essa rispetto all'ICD-9 ed infine le principali similarità e differenze ravvisabili tra la classificazione della WHO ed il DSM-III-R. Risultati - Il concetto di schizofrenia si é venuto definendo attraverso $\mathrm{i}$ contributi di autori di rilievo storico, quali Kraepelin, Bleuler e Schneider. Nella definizione dei criteri diagnostici per la schizofrenia, I'ICD-10 ha ripreso i principali criteri schneideriani relativamente ai sintomi patognomonici per questo disturbo, mentre ha ridimensionato, rispetto al DSM-III-R, l'importanza del criterio longitudinale kraepeliniano relativo al decorso, adottando una durata minima di un mese per la diagnosi e prospettando la possibilità di una prognosi non necessariamente sfavorevole. Conclusioni - Nel complesso, nell'ICD-10 si tende ad un ampliamento del concetto di schizofrenia, rispetto ad altri sistemi diagnostici più restrittivi, mentre l'enfasi posta sui sintomi schneideriani controbilancia questa tendenza e restringe i criteri di inclusione.
\end{abstract}

Parole chiave: diagnosi, classificazione, schizofrenia.

Summary. Scope - To discuss, after a description of the main attempts of classification of schizophrenic disorders made from the last century up to now, the main features of the ICD-10 classification of schizophrenia and related disorders. The main features of this chapter of the ICD-10 are then compared to the ICD-9 and DSM-III-R classifications. Results - The ICD-10 diagnostic criteria for schizophrenia require a duration of only 1 month (compared to the 6 month duration criterion of the DSM-III-R); positive symptoms of psychosis, in particular Schneiderian symptoms, are given more prominence than they are in the DSM-III-R. Moreover, it is foreseen the possibility of a favourable outcome of the disorder; this has been based on the results of several follow-up studies, including some major WHO multicentric studies showing a positive outcome in substantial proportion of patients. Conclusions - On the whole, ICD-10 broadens the diagnostic concept of schizophrenia in comparison to other diagnostic systems, whereas the emphasis placed on Schneiderian first-rank symptoms tends to overweight this tendency and makes the criteria for inclusion more narrow.

Key words: diagnosis, classification, schizophrenia.

Ricevuto il 4.3.1993 - Revisione ricevuta il 10.5.1993 - Accettato il 17.5.1993.

Indirizzo per la corrispondenza: Dr. G. de Girolamo, Division of Mental Health, WHO, 1211 Geneva 27, Switzerland.

Fax (+41) 22-791.0746.

Epidemiologia e Psichiatria Sociale, 2, 2, 1993

105 


\section{INTRODUZIONE}

La schizofrenia, almeno da Kraepelin in poi, è stata, e tuttora rimane, il vero, autentico paradigma della psichiatria e la più grande sfida ad essa posta, sul piano teorico ed operativo. La sistematizzazione nosografica di questo disturbo ha quindi rappresentato un nodo concettuale intorno al quale si sono confrontati approcci e tradizioni diverse, tradottisi in importanti ricerche volte a validare ora l'uno, ora l'altro modello concettuale. Negli ultimi anni la messa a punto dapprima del DSM-III (APA, 1980) (seguito dalla sua revisione, DSM-III-R e tra breve dal DSM-IV, di prossima introduzione) (APA, 1987; 1993) e quindi dell'ICD-10, di recente apparso (WHO, 1992), ha rappresentato un passo in avanti verso una migliore sistematizzazione nosografica dei disturbi schizofrenici.

In questo contributo, dopo un breve profilo storico relativo ai numerosi tentativi di classificazione dei disturbi schizofrenici succedutisi dal secolo scorso ad oggi, saranno descritte le principali caratteristiche della classificazione ICD-10 della schizofrenia e dei disturbi ad essa correlati, le principali novità introdotte in essa rispetto all'ICD-9 ed infine le principali similarità e differenze ravvisabili tra la classificazione della WHO ed il DSM-III-R (ed, in parte, il DSM-IV).

\section{LA DIAGNOSI DELLA SCHIZOFRENIA: UN BREVE PROFILO STORICO}

\section{La psicosi unica ed il modello kraepeliniano}

Numerose descrizioni e tentativi di classificazione dei disturbi ora definiti come schizofrenici sono stati messi a punto nella storia della psichiatria.

Nel XIX secolo prevalsero due principali approcci teorici: secondo il primo di questi due approcci, tutti i disturbi mentali erano considerati espressione di una singola entità patologica (Einheitspsychose); l'alienista tedesco Griesinger, che pubblico il suo testo fondamentale nel 1845 , fu il principale esponente di questa corrente di pensiero (Glatzel, 1990). Secondo il punto di vista alternativo, invece, i disturbi mentali dovevano essere interpretati quali entità patologiche separabili e classificabili sulla base delle differenti caratteristiche cliniche possedute da ciascuna di esse; lo psichiatra francese Morel fu l'espo- nente principale di questa scuola di pensiero e, nel 1852, denominò demence precoce un disturbo da lui ritenuto all'origine di un appiattimento dell'affettività, della comparsa di manierismi bizzarri e di una globale alterazione della personalità, che sfociava, in alcuni casi, in un vero e proprio deterioramento mentale.

Va subito sottolineato che l'antinomia tra un modello unitario di malattia mentale contrapposto ad un modello differenziato ha attraversato (e cio era già stato notato da Jaspers - 1964), come un filo rosso l'intera vicenda teorica della nosografia psichiatrica, declinandosi come opposizione tra continuità/discontinuità (in cui rientra anche il concetto dello spettro schizofrenico), ovvero categorialità/dimensionalità (per una discussione generale di queste problematiche si veda il contributo di de Girolamo in questo stesso numero) delle diverse sindromi psichiatriche (Del Pistoia \& Dalle Luche, 1993). Il modello del disturbo unitario, dopo essere stato adottato da influenti psichiatri quali Hoche nel 1912 (Stromgren, 1992), è stato, negli ultimissimi anni, pienamente ripreso da Crow (1990), il quale ha sostenuto, sulla base di evidenze eminentemente genetiche, che esisterebbe un continuum che si estende dalla depressione unipolare, attraverso il disturbo affettivo bipolare e le sindromi schizoaffettive, sino alla schizofrenia, con crescenti livelli di deficit e di gravità clinica. Tuttavia, tale modello ha ricevuto sino ad oggi solo un limitato supporto dai risultati della ricerca: anche nello studio condotto da Crow e colleghi, il Northwick Park "Functional" Psychosis Study (Johnstone et al., 1992), le psicosi affettive erano ben distinguibili, in termini di esito, da quelle schizofreniche su una importante serie di variabili, mentre solo su specifiche misure di performance psicologica, misurate con appositi test, non emergevano differenze significative tra i due principali gruppi diagnostici (schizofrenici/affettivi) basati sugli esistenti modelli di classificazione categoriale.

Dopo Morel, gli psichiatri tedeschi Kahlbaum ed Hecker, nel 1883 e nel 1871 rispettivamente, descrissero la catatonia e l'ebefrenia.

Fu tuttavia Kraepelin a gettare le fondamenta per la definizione del concetto di schizofrenia. Studiando i pazienti ricoverati negli ospedali psichiatrici al termine del XIX secolo, egli osservò che alcune forme di malattia mentale, caratterizzate da una insorgenza nella prima età adulta ed inizialmente piuttosto variabili in termini di espressività clinica, sembravano poi evolvere verso un comune esito, rappresentato da un significativo deterioramento mentale. 
Per sottolineare sia il progressivo decadimento delle capacità intellettive che l'appiattimento affettivo e la destrutturazione della personalità, da lui considerati centrali in questa condizione morbosa, Kraepelin utilizzò l'espressione (già impiegata da Morel in passato, come si è visto) di dementia praecox. Quindi nel 1896, nella $\mathrm{V}$ edizione del suo testo, per la prima volta suppose che tre condizioni, precedentemente considerate come separate, rappresentassero in realtà tre sottotipi di una singola entità morbosa, che lo stesso Kraepelin differenziò fortemente dalla psicosi maniaco-depressiva, prospettando quindi l'esistenza di due grandi classi di disturbi, a suo parere ben distinguibili (Sass, 1987). Queste tre condizioni erano rappresentate dall'ebefrenia, caratterizzata sul piano clinico da comportamenti non-finalizzati, incongrui e disorganizzati; dalla catatonia, nella quale prevalevano il negativismo ed una vistosa alterazione dello stato psicomotorio, con la presenza di uno stato stuporoso o, al contrario, di agitazione e marcato eccitamento; infine, dalla dementia paranoides, la cui fenomenologia clinica era contraddistinta da deliri di persecuzione e di grandezza. Successivamente Kraepelin aggiunse a queste tre condizioni morbose una quarta, denominata dementia praecox simplex. Inoltre separò dalla demenza precoce una nuova entità nosologica, la parafrenia, caratterizzata secondo un criterio prognostico: infatti, secondo Kraepelin, in questo disturbo, nonostante il suo carattere evolutivo, non si assisteva al decadimento della personalità. Lo psichiatra tedesco considerò la dementia praecox come una condizione contrassegnata da una prognosi invariabilmente infausta, con un decorso tendente inevitabilmente al deterioramento, nonostante che circa il $12 \%$ dei suoi pazienti mostrassero una remissione più o meno completa. Nel modello kraepeliniano, quindi, il criterio prognostico occupava un ruolo euristicamente centrale, ed era inteso come validatore ultimo della diagnosi di schizofrenia. In realtà, le ricerche successive hanno confutato tale asserzione, mettendo in evidenza una significativa eterogeneità dei decorsi e degli esiti della schizofrenia, anche quando diagnosticata con criteri restrittivi e attendibili: Warner (1991), analizzando accuratamente i 68 più importanti studi di follow-up condotti dall'inizio del secolo sino al 1980, ha riscontrato una guarigione completa all'incirca nel $20-25 \%$ dei pazienti con diagnosi di schizofrenia, ed una guarigione sul piano sociale nel $40-45 \%$ dei casi. Nelle 15 ricerche pubblicate nel decennio 1980-90, il tasso medio di guarigione è stato pari al $29 \%$, mentre quello di guarigione sociale è stato pari al $45 \%$ (de Girolamo, 1991). I ri- sultati di tali studi hanno quindi spinto Harding e Strauss (1985) a sostenere che Il concetto di omogeneità nel decorso della schizofrenia si è evoluto verso un concetto nel quale il disturbo è stato mostrare possedere (1) un decorso marcatamente eterogeneo nel tempo; (2) eterogenei livelli di capacità che attraversano differenti aree proprie del funzionamento individuale (lavoro, relazioni sociali e sintomi) nei singoli individui e (3) delle relazioni predittori-esito che suggeriscono l'esistenza e la persistenza di sistemi aperti correlati (open-linked) che costituiscono il decorso della schizofrenia così come quello di altri disturbi psichiatrici». Posizioni molto simili, nel caso della schizofrenia, sono state espresse da altri importanti autori, come ad esempio Ciompi (1988).

\section{Bleuler e Schneider}

Alcuni anni dopo Kraepelin, lo psichiatra svizzero Eugen Bleuler apportò un contributo essenziale alla concettualizzazione teorica ed all'inquadramento nosografico di questo disturbo; fu infatti Bleuler che, nel 1908, propose di designarlo per la prima volta con il nome di schizofrenia, al fine di sottolineare lo splitting (scissione) delle funzioni psichiche, che egli considerò di grande importanza nella fenomenologia del disturbo. Bleuler operò una distinzione tra i sintomi fondamentali (rappresentati innanzitutto dalla perdita dei nessi associativi, che egli considerò come la caratteristica più importante del disturbo, e quindi dall'autismo, dall'ambivalenza, dall'appiattimento dell'affettività) e quelli accessori, ritenuti non invariabilmente presenti (tra i quali egli incluse i disturbi percettivi, i deliri, i disturbi della memoria e della personalità ed i sintomi catatonici). Egli distinse anche i sintomi, dal punto di vista clinico-descrittivo, in primari, considerati quali espressione del processo della malattia, e rappresentati eminentemente da disturbi associativi e da una perturbazione dell'umore di fondo, ed in secondari, interpretati invece quali espressione di una reazione psicologica del soggetto alla malattia stessa; tra i sintomi secondari, egli annoverò l'autismo, l'ambivalenza, il deterioramento schizofrenico, i deliri ed i sintomi catatonici.

Nel complesso Bleuler ampliò significativamente il concetto di schizofrenia ed i confini nosografici del disturbo, introducendo un nuovo sottotipo (la schizofrenia simplex); inoltre egli riconobbe l'esistenza della cosiddetta "schizofrenia latente", e fece rientrare nel gruppo della schizofrenia molte sindro- 
mi sino ad allora considerate in nessun rapporto con essa, come le psicosi in personalità psicopatiche, le psicosi carcerarie e le allucinosi alcooliche.

Per quanto riguardo il decorso e la prognosi della schizofrenia, Bleuler fu dichiaratamente meno pessimista di Kraepelin e, pur ritenendo una guarigione piena un evento molto raro, sostenne che un progressivo miglioramento rappresentasse invece una evenienza abbastanza comune. Mentre Kraepelin enfatizzò la fenomenologia nella sua descrizione del disturbo, Bleuler fu più interpretativo ed il significato dei sintomi divenne più importante della loro minuziosa osservazione e classificazione (Bland \& Kolada, 1988).

Dopo Bleuler, lo psichiatra tedesco Kurt Schneider (1959) identificò un gruppo di undici sintomi che ritenne patognomonici per la schizofrenia e di raro riscontro in altri disturbi psichiatrici. Egli definì tali sintomi come "sintomi di primo rango ("first rank symptoms") (FRS) [...] non perché pensiamo che siano alla base del disturbo, ma perché essi sono di speciale valore e di particolare aiuto per apporre la diagnosi di schizofrenia... I sintomi di primo rango, tuttavia, non sempre debbono essere presenti per fare diagnosis (Schneider, 1959).

I sintomi di primo rango (voci presenti sotto forma colloquiale, voci che commentano gli atti del paziente, esperienze d'influenzamento corporeo, furto ed influenzamento del pensiero, diffusione del pensiero, percezione delirante) si identificavano largamente con i sintomi accessori di Bleuler e, sebbene non specifici della schizofrenia, erano da considerare come altamente significativi ai fini della operatività diagnostica.

I sintomi di secondo rango comprendevano invece i disturbi psicosensoriali, le intuizioni deliranti, la perplessità, i disturbi depressivi od euforici dell'umore, l'appiattimento affettivo; se presenti in numero adeguato, essi potevano essere sufficienti per la diagnosi. Nel complesso si può dire che, attraverso l'adozione di questi criteri diagnostici, Schneider operò una restrizione del concetto di schizofrenia.

I FRS di Schneider sono stati in larga misura ripresi nei moderni sistemi classificatori e diagnostici, quali i Research Diagnostic Criteria (RDC) (Spitzer et al., 1975), il DSM-III e l'ICD-10. Tuttavia, nonostante la grande importanza ad essi assegnata, la frequenza dei FRS nei pazienti con diagnosi di schizofrenia sembra variare significativamente, oscillando, nei diversi studi, da un valore minimo del $24 \%$ ad un valore massimo del $72 \%$, con una prevalenza media approssimativa del 50\% (Fenton et al., 1981). In particolare, i FRS sembrano essere meno frequenti tra i pazienti provenienti dai paesi in via di sviluppo, come fu osservato già da Wulff (1967), e come è stato confermato in uno studio prospettico nel quale un gruppo di pazienti $(\mathrm{N}=419)$ è stato valutato a Sri Lanka con la Present State Examination, ed è stato comparato ad un gruppo di pazienti inglesi $(\mathrm{N}=150)$ e canadesi $(\mathrm{N}=172)$ esaminati con lo stessa procedura standardizzata; il $25 \%$ dei pazienti nel primo gruppo presentavano dei FRS, in paragone al $54 \%$ ed al $63 \%$ nei due gruppi di pazienti occidentali (Chandrasena, 1987). Nello studio della WHO su i Determinanti di Esito dei Disturbi Mentali Gravi (DOSMED), una media del $56 \%$ dei pazienti con una diagnosi di schizofrenia nei diversi centri presentavano uno o più FRS; questi pazienti costituivano un sottogruppo caratterizzato da un elevato numero di sintomi psicotici positivi, e mostravano una marcata omogeneità sul piano clinico, pur provenendo da contesti socioculturali differenti (Jablensky et al., 1992). Inoltre, anche qui si riscontrava un differenziale nella frequenza dei FRS tra pazienti provenienti da paesi sviluppati in confronto a quelli provenienti da paesi in via di sviluppo.

La specificità dei FRS nel caso della schizofrenia è stata anche messa in dubbio, in quanto gli stessi sintomi si possono riscontrare in altri disturbi, ed in modo particolare nella mania; tra i FRS, il sintomo meno discriminante è rappresentato dalle allucinazioni in "terza persona"' (Mellor, 1982).

\section{Altri modelli nosografici}

Nei paesi scandinavi un importante contributo all'inquadramento diagnostico della schizofrenia venne fornito dallo psichiatra norvegese Langfeldt negli anni trenta (Bech, 1990). Egli distinse un gruppo di șchizofrenici processuali o nucleari, che manifestavano un esordio insidioso e un decorso verso il deterioramento, ed un gruppo "reattivo", caratterizzato da un miglior funzionamento sociale premorboso, da un esordio acuto, spesso associato ad eventi stressanti, e da una buona prognosi. Successivamente, nella tradizione psichiatrica scandinava le psicosi reattive sono state separate dalla schizofrenia nucleare e sono state denominate "psicosi schizofreniformi".

In Francia l'approccio diagnostico alla schizofrenia è stato caratterizzato da un progressivo restringimento dei criteri diagnostici e da un contemporaneo ampliamento nel numero degli stati deliranti nonschizofrenici (Pichot, 1990); tale differenziazione è 
stata accentuata enfatizzando il decorso e la prognosi sfavorevoli nel caso della schizofrenia.

In Europa, oltre agli autori sinora menzionati, molti altri hanno fornito contributi storicamente importanti per una migliore definizione del concetto di schizofrenia e per una sua sistematizzazione nosografica: tra essi vanno ricordati Otto Diem (1903), Stransky (1904), Serieux e Capgras (1909), de Clerambault (1921-24), Minkowski (1927), Kleist (1930), Binswanger (1956) (per una review del contributo più importante fornito da ciascuno di questi autori si veda Cutting \& Shepherd, 1987).

Negli Stati Uniti, invece, dagli anni '50 sino a metà degli anni ' 70 gli psichiatri hanno rivolto la loro attenzione soprattutto alla presenza dei sintomi considerati come tipicamente schizofrenici e agli aspetti difettuali caratterizzanti il decorso di questo disturbo (Warner, 1991). In particolare, gli psichiatri americani erano soliti privilegiare, nel far diagnosi, le cosiddette 4 A di Bleuler: Associazioni, Affetto, Autismo ed Ambivalenza (Andreasen \& Flaum, 1991). Tutto ciò ha condotto alla formulazione di un modello del disturbo iperinclusivo sul piano diagnostico rispetto all'approccio europeo, tradizionalmente più restrittivo, e ciò è chiaramente emerso in importanti ricerche transnazionali, rappresentate soprattutto dal progetto diagnostico anglo-statunitense (Cooper et al., 1972) e dallo studio IPSS (WHO, 1979) (per un esame approfondito dei risultati di questi due studi relativamente alla schizofrenia si rimanda all'articolo introduttivo in questo stesso numero di EPS).

In termini generali la maggior parte degli psichiatri americani faceva soprattutto riferimento, nella propria pratica diagnostica, alla presenza dei quattro sintomi bleuleriani classici, sopra elencati. A metà degli anni '70, tuttavia, si produsse un improvviso e radicale cambiamento in tali abitudini diagnostiche; cambiamento che può essere spiegato in parte con l'introduzione in terapia dei sali di litio (con la conseguente necessità di discriminare accuratamente $i$ pazienti affetti da un disturbo bipolare, candidati a tale trattamento, da quelli invece schizofrenici), in parte con le sollecitazioni provenienti da altre scuole psichiatriche, ed infine con l'introduzione di criteri più restrittivi per il rimborso delle spese sanitarie.

Cominciarono così ad emergere negli Stati Uniti i primi tentativi di standardizzazione della diagnosi di schizofrenia: tra essi va ricordato il New Haven Schizophrenia Index (Astrachan et al., 1972) ed il Sistema dei criteri flessibili di Carpenter et al. (1973). Questi ultimi autori, utilizzando i dati ottenuti nel-
l'IPSS (WHO, 1979), cercarono di individuare alcuni sintomi forniti di un elevato valore discriminativo dal punto di vista diagnostico.

Negli stessi anni un gruppo di ricercatori provenienti dalla Washington University di St. Louis elaborava i cosiddetti Feighner criteria (Feighner et al., 1972), strutturati sulla base di cinque fattori ritenuti essenziali ai fini di una soddisfacente validità diagnostica: una accurata descrizione clinica del disturbo in oggetto; eventuali indagini di laboratorio; un'accurata differenziazione da altri disturbi ottenuta attraverso l'identificazione di precisi criteri d'inclusione e di esclusione; lo studio della familiarità del disturbo; l'utilizzazione dei dati ottenuti da studi di follow-up per la valutazione dell'esito e della stabilità nel tempo della diagnosi. La diagnosi di schizofrenia, quindi, non veniva più a basarsi soltanto sulla presenza di un gruppo di sintomi discriminanti, ma anche sulla loro durata (almeno sei mesi) e su un insieme di informazioni concernenti l'adattamento premorboso, la storia familiare, il rilievo anamnestico di un disturbo affettivo o dell'uso di sostanze.

Infine, in linea con l'orientamento del gruppo di St. Louis, Spitzer et al. (1975) mettevano a punto i Research Diagnostic Criteria (RDC): in questi criteri diagnostici la durata minima del disturbo, necessaria per poter fare diagnosi, veniva ridotta a due sole settimane, e venivano introdotti nuovi criteri diagnostici differenziali con il disturbo schizoaffettivo, la schizofrenia borderline, la psicosi reattiva breve e gli stati paranoidi.

\section{LA DIAGNOSI DELLA SCHIZOFRENIA NEL DSM-III, DSM-III-R}

Nel definire i criteri diagnostici per la schizofrenia, il DSM-III ha dato particolare rilevanza ai FRS, ed ha ripreso molti dei concetti propri dei Feighner criteria e dei $R D C$, sopra menzionati. Nel complesso, nella classificazione americana sono stati adottati dei criteri restrittivi per la diagnosi di schizofrenia, privilegiando nel quadro sintomatologico i sintomi ricchi di pregnanza semeiologica, derivati a loro volta dai sintomi patognomonici di Schneider, mentre il criterio relativo alla durata (almeno sei mesi) ed alla presenza di deterioramento riflettono un avvicinamento al pensiero kraepeliniano.

La scarsa importanza attribuita ai sintomi fondamentali di Bleuler, nonostante la riproducibilità e 
l'elevata attendibilità diagnostica dei sintomi negativi (Andreasen, 1982), è stata soprattutto dettata dall'esigenza di identificare un gruppo di sintomi facilmente riconoscibili e riproducibili, e di differenziare chiaramente i vari sottotipi del disturbo, anche in rapporto alla possibilità di eventuali approcci differenziati nella terapia farmacologica.

Nel DSM-III-R (APA, 1987), quindi, sono stati introdotti alcuni cambiamenti volti ad accrescere la specificità e la sensibilità dei criteri diagnostici, operando così una ulteriore restrizione della categoria diagnostica della schizofrenia ai fini di una sua maggiore omogeneità, con un conseguente incremento dell'utilizzo, nella pratica clinica, della categoria della psicosi atipica (Fenton et al., 1988).

Di conseguenza, rispetto al DSM-III, il criterio A, composto da sei differenti items, è stato semplificato a tre items. Mentre nel DSM-III, inoltre, non era richiesta una durata minima per i sintomi psicotici acuti, nel DSM-III-R, al fine di escludere i disturbi psicotici transitori dal gruppo della schizofrenia, viene specificato che i sintomi psicotici della fase attiva indicati dal criterio $\mathrm{A}$ devono essere presenti per almeno una settimana. Questi sintomi sono divisi in tre gruppi: il primo include i deliri, le allucinazioni, l'incoerenza o il marcato allentamento dei nessi associativi, il comportamento catatonico, l'appiattimento o la grossolana inadeguatezza dell'affettività. Il secondo gruppo comprende i deliri bizzarri, deliri cioè che appaiano non plausibili ad altri membri della stessa comunità culturale. Il terzo, infine, consiste in rilevanti e specifiche allucinazioni uditive.

L'item relativo alle alterazioni dell'affettività è stato spostato, dalla posizione secondaria che aveva nel DSM-III, ad una posizione centrale all'interno degli altri items propri del criterio A, mentre, tra i sintomi prodromici e residui, è stata inclusa la "marcata perdita d'iniziativa, d'interesse o di energia”.

Il DSM-III-R, in sostanza, ha privilegiato per la schizofrenia un criterio valutativo di tipo longitudinale, definendola come un disturbo caratterizzato da un'evoluzione verso la cronicità, con un deterioramento della personalità e delle facoltà mentali (criterio $\mathrm{B})$.

Per quanto riguarda la durata, i sintomi devono essere presenti per un tempo minimo di sei mesi (criterio D), compresi il periodo prodromico e residuo.

Numerosi autori (Andreasen \& Flaum, 1991), tuttavia, hanno rilevato che l'enfatizzare, tra i criteri diagnostici per questo disturbo, un criterio di tipo longitudinale comporta il rischio di aspecificità, in quanto anche i disturbi affettivi possono presentare un decorso cronico ed un certo grado di deterioramento del funzionamento psicosociale.

Al fine di operare una diagnosi differenziale con il disturbo schizoaffettivo ed il disturbo dell'umore con aspetti psicotici, si richiede inoltre, per il criterio C, di valutare la durata totale e il periodo d'insorgenza di una sintomatologia affettiva associata.

Per quanto riguarda la differenziazione in sottotipi, il DSM-III-R ne ha riconosciuti cinque: schizofrenia paranoide, catatonica, disorganizzata, indifferenziata e residua.

Le forme di disturbo schizofrenico «senza evidenti aspetti psicotici», quali la schizofrenia latente, simplex o borderline, hanno perduto autonomia nosografica e sono incluse tra i disturbi di personalità.

Il criterio $\mathrm{E}$, infine, è volto ad escludere quadri sovrapponibili alla schizofrenia ad eziologia organica o derivanti dall'uso di sostanze.

Nel complesso, nonostante i tentativi di semplificazione operati, i criteri del DSM-III-R per poter fare diagnosi di schizofrenia sono particolarmente complessi, richiedendosi la presenza o assenza di almeno 12 differenti segni e sintomi (deliri, allucinazioni marcate, incoerenza, allentamento delle associazioni del pensiero, comportamento catatonico, appiattimento affettivo, inadeguatezza affettiva, allucinazioni verbali non legate allo stato affettivo, voci che commmentano voci che conversano, deliri bizzarri, inserzioni nel pensiero). Per questa ragione è stata enfatizzata la necessità di procedere ad una semplificazione di questi criteri, cosa che è avvenuta solo in parte nel DSM-IV (APA, 1993).

Nella quarta revisione del sistema americano, i criteri fondamentali per fare diagnosi sono rappresentati dalla presenza di almeno due tra cinque sintomi presenti per una significativa porzione di tempo durante un mese; i sintomi sono: (1) deliri; (2) allucinazioni; (3) linguaggio disorganizzato; (4) comportamento disorganizzato o catatonico; e (5) sintomi negativi. Per tanto il tempo di presenza minimo dei sintomi è stato allungato, mentre la costellazione dei sintomi necessari è stata in un certo modo semplificata. Il criterio relativo al deterioramento del funzionamento sociale è rimasto invariato, così come la continua presenza del disturbo per almeno 6 mesi. Anche gli altri criteri non sono mutati in maniera significativa. Nel complesso, quindi, il DSM-IV non comporta grandi novita per la diagnosi di schizofrenia. 


\section{DISTURBI SCHIZOFRENICI NELL'ICD-10 ED I SUOI RAPPORTI CON L'ICD-9}

Nell'ICD-9 (WHO, 1977) non erano state elaborate delle definizioni operazionali dei disturbi, necessarie per far diagnosi con soddisfacente attendibilità, ma nelle varie categorie e sottocategorie era stato inserito un glossario con funzione di guida descrittiva delle varie sindromi. La sezione "Psicosi" (290-299) del capitolo V dell'ICD-9 era pertanto suddivisa in due grandi gruppi, denominati rispettivamente «Stati Psicotici Organici» (290-294) ed "Altre Psicosi» (295-299); in quest'ultimo gruppo erano state inserite le psicosi schizofreniche, insieme alle psicosi affettive, agli stati paranoidi, alle altre psicosi non organiche ed alle psicosi con origine specifica nell'infanzia.

Nell'ICD-10, invece, nell'ambito della sezione F20-F29, denominata «Schizofrenia, Sindrome Schizotipica e Sindromi Deliranti»s sono incluse, oltre alla schizofrenia, la sindrome schizotipica, le sindromi deliranti persistenti, le sindromi psicotiche acute e transitorie, la sindrome delirante indotta e le sindromi schizoaffettive.

Per quanto riguarda in particolare la schizofrenia (F20), gli elementi nuovi introdotti nell'ICD-10 sono soprattutto relativi alla durata minima dei sintomi, che deve essere di almeno un mese, ed alla separazione delle sindromi psicotiche acute e transitorie (F23) dalla schizofrenia. L'adozione della durata minima di un mese per poter fare diagnosi di schizofrenia contraddice l'assunto che la schizofrenia debba per forza essere una condizione di lunga durata. Una durata minima dei sintomi di sei mesi è stata adottata in vari sistemi di classificazione nazionali (compreso il DSM-III); tuttavia nei due studi della WHO sulla schizofrenia (IPSS e DOSMED) (WHO, 1979; Jablensky et al., 1992), una rilevante percentuale di pazienti manifestava inequivocabili e specifici sintomi schizofrenici per più di un mese, ma per un tempo comunque inferiore a sei mesi, presentando poi un soddisfacente, se non completo, recupero. Pertanto, questa scelta è apparsa come quella più ragionevole al fine di evitare ogni implicita assunzione circa la inevitabile cronicità della schizofrenia, che viene invece concettualizzata come una sindrome che ha una molteplicità di cause (molte delle quali ancora sconosciute) ed una varietà di esiti, a loro volta dipendenti da un equilibrio di diversi fattori: genetici, neurofisiologici, sociali e culturali.

Le direttive diagnostiche prevedono poi la presenza di almeno un sintomo molto evidente compre- so tra i seguenti: (1) eco del pensiero, (2) deliri di controllo e di influenzamento, (3) voci di natura allucinatoria; e (4) deliri persistenti culturalmente idiosincratici. In alternativa, devono essere presenti sintomi appartenenti ad almeno due dei seguenti gruppi: (5) allucinazioni persistenti di qualsiasi tipo, (6) interruzioni o interpolazioni nel corso del pensiero che determinano un linguaggio incoerente, (7) comportamento catatonico, (8) sintomi negativi, come apatia, appiattimento affettivo e (9) un cambiamento costante e significativo della qualità di alcuni aspetti del comportamento personale, come perdita di interessi, ritiro sociale, ecc. E evidente, quindi, la rilevanza attribuita dal punto di vista diagnostico ai sintomi positivi di tipo Schneideriano, così come anche il rilievo assegnato ai sintomi negativi. A questo proposito va detto che numerosi studi hanno con chiarezza accertato l'attendibilità in senso diagnostico dei sintomi negativi, la loro interna consistenza e l'elevato grado di intercorrelazione tra essi, l'associazione con dei criteri di validazione indipendente (quali ad esempio l'esistenza di anormalità sul piano neuro-anatomico) ed una elevata stabilità temporale dei sintomi stessi (Andreasen \& Flaum, 1991).

L'ICD-9 non escludeva la diagnosi di schizofrenia, qualunque fosse il danno organico concomitante; nell'ICD-10 viene invece specificata l'incompatibilità di questa diagnosi in caso di «malattia cerebrale manifesta o negli stati d'intossicazione o astinenza da droghe».

La sindrome schizotipica (F21) è stata inclusa nell'ICD-10 nella sezione $F 2$ in virtù delle manifestazioni cliniche, della storia familiare e del decorso; essa è descritta come una condizione caratterizzata da un comportamento eccentrico e da anomalie del pensiero e dell'affettività, che somigliano a quelle osservate per la schizofrenia, ma che non sono tali da soddisfare i criteri diagnostici per tale disturbo. In essa è stata inclusa il sottotipo della «schizofrenia latente», per come esso era definito nell'ICD-9. Nelle direttive diagnostiche ne è comunque sconsigliato l'impiego per un uso «esteso, perché questo disturbo non è chiaramente delimitato dalla schizofrenia simplex, nè dai disturbi schizoidi o paranoidi di personalità» ed, a differenza del DSM-III-R, è sottolineata l'importanza della durata continua od episodica dei sintomi per almeno due anni per poter fare questa diagnosi. Va sottolineato che questa categoria diagnostica è stata, da lungo tempo, all'origine di controversie tra differenti autori per quanto riguarda la sua collocazione nosografica: tuttavia studi genetici (Baron et al., 1985), familiari (Kendler et al., 
1985; Torgensen, 1985) e neurofisiologici sembrano confermare l'esistenza di un continuum tra schizofrenia e disturbo schizotipico allorquando si considerano le caratteristiche del disturbo tradizionalmente riportate, quali isolamento sociale, sospettosità, freddezza ed eccentricità.

Le sindromi psicotiche acute e transitorie (F23) rappresentano una categoria di nuova introduzione nell'ICD-10: i dati che emergono dall'esperienza clinica e dalle ricerche di tipo transculturale sembrano infatti dimostrare che, soprattutto nei paesi in via di sviluppo, si riscontra un rilevante numero di psicosi acute con «esordio improvviso, evoluzione breve nel giro di poche settimane o perfino di pochi giorni, ed esito favorevole" (Wig \& Parhee 1989).

In generale, nell'ICD-10 tre condizioni sono considerate necessarie per poter fare diagnosi di sindrome psicotica acuta e transitoria: (a) l'esordio acuto (entro le due settimane), (b) la presenza di sindromi tipiche, quali una condizione rapidamente mutevole e variabile, definita "polimorfa» e la presenza di tipici sintomi schizofrenici; e (c) la presenza di un fattore stressante acuto precedente l'esordio della sintomatologia entro un tempo di due settimane. Tuttavia viene anche precisato che un gran numero di sindromi psicotiche acute insorge senza fattori stressanti associati, per cui è possibile registrare sia l'assenza che la presenza dello stress.

La presenza di sintomi prodromici o di alterazioni del livello funzionale dell'individuo non sono invece da considerarsi rilevanti per quanto riguarda la durata della sindrome.

Nell'ambito di questo gruppo diagnostico, vengono quindi riconosciuti differenti sottotipi, quali: (a) la sindrome psicotica acuta polimorfa senza sintomi schizofrenici, la cui diagnosi viene fatta in presenza di un esordio acuto, di vari tipi di deliri ed allucinazioni mutevoli in tipo ed intensità da giorno a giorno o nello stesso giorno e di uno stato emozionale altrettanto variabile; (b) la sindrome psicotica acuta polimorfa con sintomi schizofrenici, nella cui fenomenologia compaiono anche sintomi della serie schizofrenica; (c) la sindrome psicotica acuta schizofrenosimile, che pur presentando una sintomatologia stabile, che rispecchia quella schizofrenica, ha una durata inferiore ad un mese; (d) l'altra sindrome psicotica acuta prevalentemente delirante, nella quale $i$ sintomi principali sono rappresentati da deliri ed allucinazioni relativamente stabili, ma la cui durata (inferiore ad un mese) differenzia questa condizione dalla sindrome delirante persistente, in cui deliri ed allucinazioni hanno una durata superiore a tre mesi; (e) le altre sindromi psicotiche acute e transitorie ed infine (f) la sindrome psicotica acuta e transitoria non specificata.

Alcuni dei disturbi inclusi in questo gruppo sono assimilabili alle psicosi cicloidi (Perris, 1974) ed alla bouffeè delirante (Pull et al., 1988). Sebbene il concetto di bouffeè delirante sia tradizionalmente stato più restrittivo rispetto a quello di psicosi cicloide, disturbo che comprendeva importanti e specifici disturbi della motilità e dell'affettività, entrambi questi quadri clinici erano caratterizzati da un estrema variabilità dell'espressione sintomatologica e presentavano un certo numero di caratteristiche simili: un esordio acuto, una durata breve, una buona prognosi, un decorso recidivante, la presenza di deliri ed allucinazioni, di elementi confusionali di vario grado, infine di depressione/eccitazione. L'ICD-10, quindi, ha conservato i lineamenti tradizionali di questi due disturbi e li ha assimilati alla sindrome acuta polimorfa senza (o con) sintomi schizofrenici. Nell'ICD-9, invece, la psicosi cicloide era considerata equivalente alla psicosi schizoaffettiva, mentre la bouffeè delirante rientrava nella reazione paranoide acuta senza trovare in essa una diretta equivalenza (Pichot, 1990).

Infine, le sindromi schizoaffettive (F25), che nell'ICD-9 erano classificate come un sottotipo della schizofrenia, nell'ICD-10 vengono considerate come una categoria a sè stante, insieme ai relativi sottotipi (maniacale, depressivo, misto, di altro tipo e non specificato). La decisione di inserire le sindromi schizoaffettive nella sezione F2 è stata presa dopo i risultati dei field trials, condotti con la versione provvisoria dell'ICD-10 del 1987, e riflette gli orientamenti dominanti nella comunità psichiatrica internazionale, volti a sottolineare la maggiore contiguità di questo disturbo con le sindromi schizofreniche piuttosto che con quelle affettive. Le direttive diagnostiche richiedono pertanto, per poter far diagnosi di sindrome schizoaffettiva, la presenza di sintomi schizofrenici ed affettivi simultanei e di simile intensità durante lo stesso episodio.

\section{ICD-10, DSM-III-R E DSM-IV: SIMILARITÀ E DIFFERENZE NOSOGRAFICHE E DIAGNOSTICHE}

La tabella I mostra le categorie diagnostiche relative ai disturbi schizofrenici contenute nell'ICD-10, nel DSM-III-R e nel DSM-IV. Come si può vedere, 
Tabella I. - Classificazione dei disturbi schizofrenici nell'ICD-10, nel DSM-III-R e nel DSM-IV.

\begin{tabular}{|c|c|c|}
\hline ICD-10 & DSM-III-R & DSM-IV \\
\hline Schizofrenia & Schizofrenia & Schizofrenia \\
\hline Paranoide & Paranoide & Paranoide \\
\hline Ebefrenica & Disorganizzata & Disorganizzata \\
\hline Catatonica & Catatonica & Catatonica \\
\hline Indifferenziata & Indifferenziata & Indifferenziata \\
\hline Residua & Residua & Residua \\
\hline \multicolumn{3}{|l|}{ Depressione post-schizofrenica } \\
\hline \multicolumn{3}{|l|}{ Simplex } \\
\hline \multicolumn{3}{|l|}{ Altra } \\
\hline \multicolumn{3}{|l|}{ Non specificata } \\
\hline \multicolumn{3}{|l|}{$\begin{array}{l}\text { Sindrome } \\
\text { schizotipica }\end{array}$} \\
\hline $\begin{array}{l}\text { Sindrome } \\
\text { delirante persistente }\end{array}$ & Disturbo delirante (paranoide) & Disturbo delirante \\
\hline \multicolumn{3}{|l|}{ Sindromi psicotiche acute e transitorie } \\
\hline \multicolumn{3}{|l|}{$\begin{array}{l}\text { Sindrome psicotica acuta polimorfa senza } \\
\text { sintomi schizofrenici }\end{array}$} \\
\hline $\begin{array}{l}\text { Sindrome psicotica acuta polimorfa con sin- } \\
\text { tomi schizofrenici }\end{array}$ & Psicosi reattiva breve & Disturbo psicotico breve \\
\hline Sindrome psicotica acuta schizofrenosimile & Disturbo schizofreniforme & Disturbo schizofreniforme \\
\hline \multicolumn{3}{|l|}{$\begin{array}{l}\text { Altra sindrome psicotica acuta prevalente- } \\
\text { mente delirante }\end{array}$} \\
\hline Sindrome delirante indotta & Disturbo psicotico indotto & Disturbo psicotico condiviso \\
\hline \multirow[t]{2}{*}{ Sindromi schizoaffettive } & Disturbi schizoaffettivi & Disturbi schizoaffettivi \\
\hline & & $\begin{array}{l}\text { Disturbo psicotico dovuto ad una } \\
\text { condizione medica generale } \\
\text { - con deliri } \\
\text { - con allucinazioni }\end{array}$ \\
\hline \multicolumn{3}{|l|}{ Altre sindromi psicotiche non organiche } \\
\hline Psicosi non organica non specificata & Disturbo psicotico non altrimenti specificato & $\begin{array}{l}\text { Disturbo psicotico non altrimenti } \\
\text { specificato }\end{array}$ \\
\hline
\end{tabular}

la suddivisione dell'ICD-10 in sottotipi, relativamente alla schizofrenia di tipo paranoide, catatonico, indifferenziato, ebefrenico e residuo è simile a quella riportata nel DSM-III-R e nel DSM-IV. A questo proposito va ricordato che McGlashan \& Fenton (1991), in una review relativa alla classificazione della schizofrenia in sottotipi, hanno concluso che i dati disponibili consentono di sostenere la validità dei sottotipi esistenti, in particolare della forma paranoide; la validità delle forme ebefrenica ed indifferenziata è altrettanto dimostrata, seppur in maniera meno definitiva. È interessante rilevare come i due au- tori sostengano che $\mathrm{i}$ dati disponibili supportano la validità della forma simplex, inclusa nell'ICD-10 ma non nel DSM-III-R o nel DSM-IV.

\section{Schizofrenia (F20)}

Le principali differenze tra la classificazione della schizofrenia nell'ICD-10 e nel DSM-III-R sono rappresentate dalla durata minima dei sintomi (rispettivamente un mese e sei mesi), e dal maggiore peso attribuito nell'ICD-10 ai FRS di Schneider. Nel- 
l'ICD-10, inoltre, l'importanza dei sintomi negativi è enfatizzata ed essi vengono chiaramente identificati. In questo senso, mentre la maggior importanza assegnata nell'ICD-10 ai FRS tende a restringere i criteri per la diagnosi, la minor durata richiesta, il valore attribuito ai sintomi negativi e l'inclusione della schizofrenia simplex portano all'inclusione di un maggior numero di pazienti in questa categoria diagnostica.

Nell'ICD-10 il periodo prodromico proprio del disturbo è descritto in maniera analoga al DSMIII-R, ma non è considerato di ausilio diagnostico e non è incluso nel tempo di durata previsto per fare diagnosi, che è di almeno un mese.

Una differenza importante tra i due sistemi classificatori, che ha, come si è visto, importanti implicazioni sul piano concettuale, è che nell'ICD-10, a differenza del DSM-III-R, viene sottolineato che il decorso della schizofrenia è «variabile e non è inevitabilmente cronico o invalidante».

Se la sintomatologia presente corrisponde al quadro descritto per la schizofrenia, ma la sua durata è inferiore a un mese, l'orientamento diagnostico proprio dell'ICD-10 è in favore della sindrome psicotica acuta schizofrenosimile (che può, eventualmente, essere codificata come diagnosi provvisoria). L'adozione della durata minima di un mese nell'ICD-10 può quindi portare all'inclusione, in questa categoria diagnostica, di casi diagnosticati invece, secondo il DSM-III-R, come disturbo schizofreniforme.

Anche nell'ICD-10, come nella classificazione americana, in presenza di sintomi affettivi accentuati si esclude la diagnosi di schizofrenia, ad eccezione dei casi in cui questa è cronologicamente insorta prima.

Nel complesso, quindi, i due testi mostrano delle significative differenze, al punto che Andreasen \& Flaum (1991) hanno sostenuto che, mentre secondo la definizione dell'ICD-10, si delinea il quadro di un disturbo caratterizzato da un periodo psicotico marcato relativamente breve, il DSM-III-R fa trasparire l'immagine di un disturbo meno caratterizzato dal punto di vista della sintomatologia psicotica ma maggiormente tendente alla cronicità.

Sino ad oggi un solo studio ha comparato la affidabilità e la facilità d'uso dell'ICD-10 e del DSMIII-R in un piccolo gruppo di psichiatri che, in coppia, hanno valutato dal punto di vista diagnostico 60 pazienti (Mellsop et al., 1991). I due sistemi hanno mostrato una elevata concordanza per la categoria generale di schizofrenia, ma non per i sottotipi, come il paranoide e l'ebefrenico.

\section{Sindrome schizotipica (F21)}

Questa categoria corrisponde al «disturbo di personalità» schizotipico del DSM-III-R e del DSM-IV; nel manuale americano è confluita in questa categoria diagnostica anche la schizofrenia simplex, che permane invece nell'ICD-10 come sottotipo della schizofrenia.

\section{Sindromi psicotiche acute e transitorie (F23)}

Nel DSM-III-R e nel DSM-IV questi disturbi sono compresi nelle categorie della psicosi reattiva breve (chiamato nel DSM-IV disturbo psicotico breve) e del disturbo schizofreniforme con buona prognosi, a loro volta inclusi nel capitolo relativo ai disturbi psicotici non classificati altrove. In particolare, nel caso della psicosi reattiva breve del DSM-III-R, viene prevista la presenza di sintomi della serie schizofrenica associati a stati variabili di agitazione emotiva, perplessità e confusione. Questo quadro nosologico corrisponde quindi, per alcuni tratti, ad entrambe le sindromi psicotiche acute polimorfe con e senza sintomi schizofrenici, nonostante in queste ultime non sia necessaria la presenza di fattori stressanti e quindi possa venir meno il carattere reattivo.

Nell'ICD-10 il termine schizofreniforme non è stato invece utilizzato perché ritenuto confusivo dopo la sua applicazione negli ultimi decenni a "vari differenti concetti clinici», e dopo l'impiego che ne è fatto nel DSM-III-R, cioé di una categoria intermedia caratterizzata da una durata della sintomatologia schizofrenica inferiore a sei mesi; infatti l'introduzione della durata di un mese dei sintomi psicotici come criterio diagnostico per la schizofrenia, e l'inserimento nel gruppo delle sindromi psicotiche acute e transitorie dei disturbi aventi un profilo descrittivo sovrapponibile al disturbo schizofreniforme ne rende superflua una trattazione autonoma nell'ICD-10.

Pertanto, sebbene non esista una chiara equivalenza tra i due sistemi diagnostici a questo proposito, le indicazioni fornite nell'ICD-10 a coloro che desiderano usare questo termine sono di orientarsi verso altre categorie diagnostiche, in particolare la schizofrenia di altro tipo e la sindrome psicotica acuta schizofrenosimile.

\section{Sindrome schizoaffettiva (F25)}

Il DSM-III-R ha collocato il disturbo schizoaffettivo nel gruppo dei «Disturbi Psicotici Non Classifi- 
cati Altrove» (295.70), e, rifacendosi ai criteri di Welner e ai RDC, ha delineato dei criteri diagnostici restrittivi, nei quali viene privilegiata la relazione temporale rispetto alla comparsa dei sintomi schizofrenici ed affettivi: i sintomi delle due serie devono manifestarsi contemporaneamente (criterio A), e «in un episodio del disturbo i sintomi schizofrenici devono permanere per almeno due settimane senza rilevanti sintomi affettivi» (criterio B).

«Se la durata totale di tutti gli episodi di alterazione dell'umore è breve rispetto alla durata totale del disturbo, oppure il disturbo dell'umore si verifica soltanto durante la fase residua» si pone diagnosi di schizofrenia, mentre in assenza delle condizioni che rispettano il criterio B si pone diagnosi di Disturbo dell'Umore con Aspetti Psicotici.

Nel DSM-III-R, pertanto, viene fornito un giudizio clinico trasversale e longitudinale, mentre nell'ICD-10 prevale un giudizio diagnostico cross-sezionale.

Nel DSM-III-R l'inserimento nei disturbi psicotici NCA del disturbo schizoaffettivo, che precedentemente era stato considerato una variante della schizofrenia, rispecchia il fatto che questa categoria è utilizzata, nella classificazione americana, come categoria di riserva per quelle condizioni che non soddisfano i criteri per la schizofrenia o per i disturbi dell'umore.

\section{RISULTATI DEI FIELD TRIALS DELL'ICD-10}

Nei field trials relativi all'ICD-10 (per una descrizione più generale si rinvia al contributo precedente), i disturbi inclusi in questo capitolo hanno ottenuto un valore di Kappa pari a 0,82: tale valore rappresentava quello più elevato tra tutti i vari capitoli, ad eccezione delle sindromi e disturbi comportamentali associati ad alterazioni delle funzioni fisiologiche e a fattori somatici (Sartorius et al., 1993). Tuttavia, allorquando si trattava di specificare il disturbo in questione all'interno del capitolo F2, utilizzando categorie diagnostiche a quattro codici, i valori di Kappa variavano marcatamente, andando da un massimo di 0,73 per la schizofrenia paranoide ad un minimo di 0,30 per la categoria della sindrome psicotica acuta e transitoria altra (F238). Nel caso di quest'ultima categoria, il basso valore di accordo diagnostico riscontrato può essere spiegato con la sua aspecificità, dal momento che in essa dovrebbero essere in- clusi solo i casi che non soddisfano i criteri diagnostici per una diagnosi più precisa. Inoltre altri due disturbi, rappresentati dalla sindrome schizotipica (F21) e dalla sindrome psicotica acuta schizofrenosimile, manifestavano un valore di Kappa quasi altrettanto basso; in questo caso, tale risultato ha fornito lo spunto per una revisione del testo dell'ICD-10, volta a meglio precisare i criteri diagnostici necessari per questi due disturbi.

\section{LA RILEVANZA DEI PROBLEMI DIAGNOSTICI PER LA RICERCA EPIDEMIOLOGICA E PER LA CLINICA}

Nell'ambito della ricerca epidemiologica, e della pratica clinica altrettanto, la rilevanza centrale dei problemi diagnostici discussi sopra dipende dal fatto che cambiamenti intervenuti nella classificazione $e$ nei criteri diagnostici per la schizofrenia possono modificare significativamente i tassi di prevalenza ed incidenza del disturbo rilevati nei vari studi, ed avere implicazioni prognostiche e terapeutiche diverse. Sono già stati descritti in dettaglio, nel precedente contributo, $\mathrm{i}$ risultati ottenuti nel progetto diagnostico anglo-statunitense, nell'IPSS e nello studio di Loranger, che sono paradigmatici a questo proposito. A ciò va aggiunto che più di dieci studi comparativi, effettuati per mettere a confronto una dozzina di diverse definizioni operazionali della diagnosi di schizofrenia, hanno dimostrato consistentemente come $i$ tassi di prevalenza e di incidenza riguardanti questo disturbo varino significativamente a seconda dei criteri diagnostici adottati (Sass, 1987). In generale, le principali differenze tra i vari sistemi diagnostici concernono la scelta dei sintomi selezionati per fare diagnosi, la struttura degli algoritmi diagnostici, la scelta di una durata minima della sintomatologia e la diversa valutazione dei sintomi affettivi presenti. In uno dei più importanti studi comparativi, Endicott et al. (1982) misero a confronto sei gruppi di criteri diagnostici (New Haven, Carpenter, Feighner, Taylor and Abrams, DSM-III and RDC) in un campione di 108 pazienti. I sei sistemi risultarono paragonabili in termini di affidabilità, ma la frequenza dei casi diagnosticati come schizofrenici oscillò dal $4 \%$ al $26 \%$ a seconda del sistema impiegato; i RDC, il sistema di Carpenter ed il DSM-III risultarono i sistemi diagnostici più omogenei. Le principali variabili alla base di queste differenze nel numero di casi dia- 
gnosticati sembrarono essere rappresentate da: (a) $i$ criteri d'esclusione dei pazienti con disturbi affettivi, (b) i criteri di durata minima adottati e (c) il grado di specificazione di ogni item. In un altro studio, Brockington et al. (1978) rilevarono che, comparando 10 differenti set di criteri diagnostici, la percentuale di casi di schizofrenia, in un gruppo di 119 pazienti al loro primo ricovero, variava dal $3 \%$ al $38 \%$ a seconda del criterio impiegato: RDC, CATEGO, Carpenter and Langfeldt manifestavano una buona concordanza, mentre altri apparivano troppo restrittivi (Feighner criteria), ed altri ancora (Schneider) erano deboli predittori di esito sia clinico che sociale.

In aun altro studio, McGlashan (1984), rianalizzando circa 400 casi di pazienti trattati a Chestnut Lodge ed impiegando 4 criteri diagnostici (New Haven RDC, Feighner e DSM-III) trovò nel complesso una buona concordanza tra questi tre ultimi.

Helzer (1981), quindi, utilizzando i dati relativi a St. Louis nell'ambito dell'Epidemiological Catchment Area Program (ECA), ha messo in evidenza una differenza di cinque volte nelle stime di prevalenza per la schizofrenia ottenute impiegando i criteri Feighner, i RDC o il DSM-III; è interessante notare che una differenza della stessa ampiezza (con tassi di prevalenza fino a cinque volte maggiori) fu rilevata anche da Dunham (1965) in una review sull'epidemiologia della schizofrenia; questo dato dimostra pertanto che differenze di questa ampiezza nei tassi di prevalenza della schizofrenia potrebbero essere interamente definizionali.

Per finire, Kuriansky et al. (1974) hanno dimostrato che, mentre più dell' $80 \%$ dei pazienti psichiatrici ricoverati in un ospedale psichiatrico di New York durante i primi anni ' 50 erano diagnosticati come schizofrenici, nella decade precedente la percentuale era inferiore al $40 \%$, e questo mutamento era in buona parte da ascrivere ai mutamenti intervenuti nella prassi diagnostica relativamente al disturbo in questione.

\section{NOTE CONCLUSIVE}

Nella definizione dei criteri diagnostici per la schizofrenia, l'ICD-10 ha ripreso i principali criteri schneideriani relativamente ai sintomi patognomonici per questo disturbo, mentre ha ridimensionato, rispetto al DSM-III-R, l'importanza del criterio longi- tudinale kraepeliniano relativo al decorso, adottando una durata minima di un mese per la diagnosi e prospettando la possibilità di una prognosi non necessariamente sfavorevole. Nel complesso, quindi, nell'ICD-10 si tende ad un ampliamento del concetto di schizofrenia, rispetto ad altri sistemi diagnostici più restrittivi, mentre l'enfasi posta sui sintomi schneideriani controbilancia questa tendenza e restringe i criteri di inclusione.

Nello stesso capitolo è stata inoltre introdotta la sindrome schizotipica, ed è stato notevolmente esteso il gruppo delle sindromi psicotiche acute e transitorie. Le ricerche future dovranno dimostrare la affidabilità e validità diagnostica di queste scelte.

\section{BIBLIOGRAFIA}

American Psychiatric Association (1980). Diagnostic and Statistical Manual of Mental Disorders (DSM III) (3rd ed.). American Psychiatric Association: Washington.

American Psychiatric Association (1987). Diagnostic and Statistical Manual of Mental Disorders (DSM III) (3rd ed. revised). American Psychiatric Association: Washington.

American Psychiatric Association (1993). DSM-IV Draft Criteria. American Psychiatric Association: Washington.

Andreasen N.C. (1982). Negative symptoms in schizophrenia: definition and reliability. Archives of General Psychiatry 39, 784-788.

Andreasen N.C. \& Flaum M. (1991). Schizophrenia: the characteristic symptoms. Schizophrenia Bulletin 17, 27-49.

Astrachan B.M., Harrow M., Adler D., Brauer L., Schwartz C. \& Tucker G. (1972). A checklist for the diagnosis of schizophrenia. British Journal of Psychiatry 121, 529-536.

Baron M., Gruen R., Rainer J.D., Kane J., Asnis L \& Lord S. (1985). A family study of schizophrenic and normal control probands. Implications for the spectrum concept of schizophrenia. American Journal of Psychiatry 142, 447-455.

Bech P. (1990). Diagnostic and classification tradition of mental disorders in the 20th century in Scandinavia. In Sources and Traditions of Classification in Psychiatry. (ed. N. Sartorius, A. Jablensky, D.A. Regier, J.D. Burke and R.M.A. Hirshfeld), pp. 153-70. Hogrefe \& Huber: Berna.

Bland R.C. \& Kolada J. (1988). Diagnostic issues and current criteria for schizophrenia. In Handbook of Schizophrenia. Vol.3, Nosology, Epidemiology and Genetics (ed. M.T. Tsuang and J.C. Simpson), pp. 1-25. Elsevier: Amsterdam.

Brockington I.F., Kendell R.E. \& Leff J.P. (1978). Definitions of schizophrenia, concordance and prediction of outcome. Psychological Medicine 8, 387-398.

Carpenter W.T., Strauss J.S. \& Bartko J. (1973). Flexible system for the diagnosis of schizophrenia. Report from the World Health Organization Pilot Study of Schizophrenia. Science $182,1275-1278$.

Chandrasena R. (1987). Schneider's first rank symptoms, An international and interethnic comparative study. Acta Psychiatrica Scandinavica 76, 574-578. 
Ciompi L. (1988). Learning from outcome studies. Toward a comprehensive biological-psychosocial understanding of schizophrenia. Schizophrenia Research 1, 373-384.

Cooper J.E., Kendell R.E., Gurland B.J., Sharpe L., Copeland J.R.M. \& Simon R. (1972). Psychiatric Diagnosis in New York and London: A Comparative Study of Mental Hospital Admissions (Maudsley Monograph No. 22). Oxford University: London,

Crow T.J. (1990). Nature of the genetic contribution to psychotic illness - a continuum viewpoint. Acta Psychiatrica Scandinavica $81,401-408$.

Cutting J. \& Shepherd M. (1987). The Clinical Roots of the Schizophrenia Concept. Cambridge University Press: Cambridge.

de Girolamo G. (1991). Schizofrenia: dal mito kraepeliniano ai modelli psicosociali. In Guarigione e Schizofrenia (ed. R. Warner), pp. 15-59. Feltrinelli: Milano.

Del Pistoia L. \& Dalle Luche R. (1993). La psicosi unica tra paradigmi nosografici non categoriali e strutture di senso in psicopatologia. In Psichiatria nella Comunità: Cultura e Pratica (ed. F. Asioli, A. Ballerini e G. Berti Ceroni), pp. 13-35. Bollati Boringhieri: Torino.

Dunham H.W. (1965). Community and Schizophrenia: an Epidemiological Analysis. Wayne State University Press: Detroit.

Endicott J.N., Fleiss J., Cohen J., Janel W. \& Simon R. (1982). Diagnostic criteria for schizophrenia. Archives of General Psychiatry 39, 884-889.

Feighner J.P., Robins E., Guze S.B., Woodruff R.A. \& Winokur G. (1972). Diagnostic criteria for use in psychiatric research. Archives of General Psychiatry 26, 57-63.

Fenton W.S., Mosher L.R. \& Matthews S.M. (1981). Diagnosis of schizophrenia, a critical review of current diagnostic systems. Schizophrenia Bulletin 7, 452-476.

Fenton W., McGlashan T. \& Heinssen R.K. (1988). A comparison of DSM-III and DSM-III-R schizophrenia. American Journal of Psychiatry 145, 1446-1449.

Glatzel J. (1990). Psychiatric diagnosis in the German-speaking countries. In Sources and Traditions of Classijication in Psychiatry (ed. N. Sartorius, A. Jablensky, D.A. Regier, J.D. Burke and R.M.A. Hirshfeld), pp. 58-92. Hogrefe \& Huber: Berna.

Harding C. M. \& Strauss J. S. (1985). The course of schizophrenia: an evolving concept. In Controversies in Schizophrenia (ed. M. Alpert), pp. 339-353. Guilford: New York.

Helzer J.E., Robins L.N., Croughan J.L. \& Welner A. (1981). Renard diagnostic interview. Archives of General Psychiatry 38, 393-398.

Jablensky A., Sartorius N., Ernberg G., Anker M., Korten A., Cooper J.E,, Day R. \& Bertelsen A. (1992). Schizophrenia, Manifestations, Incidence and Course in Different Cultures. A World Health Organization Ten-Country Study. Psychological Medicine Monograph Supplement 20. Cambridge University Press: Cambridge

Jaspers K. (1964). Psicopatologia Generale. Il Pensiero Scientifico: Roma

Johnstone E.C., Frith C.D., Crow T.J., Owens D.G.C., Done D.J., Baldwin E.I. \& Charlette A. (1992). The Northwick Park «Functional» Psychosis Study: diagnosis and outcome. Psychological Medicine 22, 331-346.

Kendler K.S., Masterson CC. \& Davis KL. (1985). Psychiatric ilIness in first-degree relatives of patients with paranoid psychosis, schizophrenia and medical illness. British Journal of Psychiatry 147, 524-531.
Kuriansky J.B., Denning W.E. \& Gurland B.J. (1974). On trends in the diagnosis of schizophrenia. American Journal of Psychiatry 131, 402-408.

McGlashan T.H. (1984). Testing four diagnostic systems for schizophrenia. Archives of General Psychiatry 41, 141-144.

McGlashan T. \& Fenton W. (1991). Clinical subtypes for schizophrenia. Schizophrenia Bulletin 17, 609-632.

Mellor C.S. (1982). The present status of first-rank symptoms. British Journal of Psychiatry 140, 423-424.

Mellsop G.W., Thomas C.S., Ellis P.M., Purdie G., Crawshaw J. \& Mendis M. (1991). Reliability of the draft diagnostic criteria for research of ICD-10 in comparison with ICD-10 and DSMIII-R. Acta Psychiatrica Scandinavica 84, 332-335.

Perris C. (1974). A study of cycloid psychoses. Acta Psychiatrica Scandinavica, Supplementum 253.

Pichot P. (1990). The diagnosis and classification of mental disorders in the French-speaking countries, background, current values and comparison with other classifications. In Sources and Traditions of Classification in Psychiatry (ed. N. Sartorius, A. Jablensky, D.A. Regier, J.D. Burke and R.M.A. Hirshfeld), pp. 7-57. Hogrefe \& Huber: Berna.

Pull C.B., Pull M.C. \& Pichot P. (1988). The French approach to psychiatric classification. In International Classification in Psychiatry (ed. J.E. Mezzich and M. von Cranach), pp. 37-47. Cambdridge University Press: Cambridge.

Sartorius N., Kaelber C., Cooper J., Roper M., Rae D., Gulbinat W., Ustun B. \& Regier D (1993). Progress towards achieving a common language in psychiatry: results from the field trial of the clinical guidelines accompanying the WHO classification of mental and behavioural disorders in the ICD-10. Archives of General Psychiatry 50, 115-124.

Sass H. (1987). The classification of schizophrenia in the different diagnostic systems. In Search for the Causes of Schizophrenia (ed. H.Hafner, W.F. Gattaz and W. Janzarik), pp.19-43. Springer-Verlag: Berlin.

Schneider K. (1959). Klinische Psychopathologie. Thieme: Stuttgart.

Spitzer R.L., Endicott, J. \& Robins, E. (1975). Research Diagnostic Criteria. New York State Psychiatric Institute: New York.

Stromgren E. (1992). The concept of schizophrenia: the conflict between nosological and symptomatological aspects. Journal of Psychiatric Research 26, 237-246.

Torgensen S. (1985). Relationship of schizotypal personality disorder to schizophrenia. Schizophrenia Bulletin 11, 554-564.

Warner R. (1991). Schizofrenia e Guarigione. Feltrinelli: Milano.

Wig N.N. \& Parhee R. (1989). Acute and transient psychoses: a view from the developing countries. In International Classification in Psychiatry, Unity and Diversity (ed. J.E. Mezzich, and $M$. von Cranach). Cambridge University Press: Cambridge.

World Health Organization (1977). International Classification of Diseases, 9th Revision. WHO: Geneva.

World Health Organization (1979). The International Pilot Study of Schizophrenia. Wiley Chichester.

World Health Organization (1992). International Classification of Diseases, 10th Revision. WHO: Geneva. Ed. it.: Decima Revisione della Classificazione Internazionale delle Sindromi e dei Disturbi Psichici e Comportamentali. Descrizioni Cliniche e Direttive Diagnostiche (ed. D. Kemali, M. Maj, F. Catapano, S. Lobrace e L. Magliano). Masson: Milano.

Wulff E. (1967). Psichiatrischer Bericht aus Vietnam. In Beitrage suŕ vergleichenden Psychiatrie (ed. N. Petrilowitsch). Karger: Basel. 
Gaspare Vella - Danilo Solfaroli Camillocci

\section{Né CON TE, NÉ SENZA di TE. \\ La coppia in stallo}

220 pagine. Lire 30.000

\section{Introduzione}

Caratteristiche generali della coppia in stallo

Le mosse e le contromosse

Lo stallo per opposizione

Lo stallo per raggiro

La comunicazione nella coppia in stallo

La genesi della relazione di stallo

L'interferenza della famiglia d'origine

L'inibizione della forza

Le mosse che portano alla situazione di stallo

L'inconcludenza e la fiducia

\section{Dalla diade alla triade: la pania}

\section{Le linee terapeutiche}

La prima fase: la terapia con la famiglia

Bloccare le triangolazioni

Impedire intrusioni dei figli

Distinguere il coniugale dal genitoriale

Svelare i livelli implicati nel discorso

Rilevare apertamente le triadi e le coalizioni

Portare allo scoperto e far risperimentare il piacere dello scontro diretto

Sottolineare il senso positivo delle provocazioni

La seconda fase della terapia: l'esposizione della storia e il passaggio alla terapia di coppia

La terza fase: la terapia della coppia

Rilevare e dare un significato alle triangolazioni, che tendono a verificarsi anche in assenza dei figli

Dalle recriminazioni alle richieste

Far riemergere la passione

Spingere ad affrontare con chiarezza i temi dell'oggettività coniugale

\section{Problemi ancora aperti}

Paralipomeni

\section{Pensiero Scientifico Editore}

via Bradano 3/c - 00199 Roma

Tel. (06) 86207158/59/68/69 